\title{
Priming of luminance-defined motion direction in visual search
}

\author{
Árni Kristjánsson, ANdri BJarnason, Árni Bragi HJaltason, \\ AND BRYNDÍS GYĐA STEFÁNSDótTIR \\ University of Iceland, Reykjavik, Iceland
}

\begin{abstract}
Features that we have recently attended to strongly influence how we allocate visual attention across a subsequently viewed visual scene. Here, we investigate the characteristics of any such repetition effects during visual search for Gabor patch targets drifting in the odd direction relative to a set of distractors. The results indicate that repetition of motion direction has a strong effect upon subsequent allocation of attention. This was the case for judgments of a target's presence or absence, of a target's location, and of the color of a target drifting in the odd direction. Furthermore, distractor repetition on its own can facilitate search performance on subsequent trials, indicating that the benefits of repetition of motion direction are not confined to repetition of target features. We also show that motion direction need not be the target-defining dimension throughout a trial block for motion priming to occur, but that priming can build up with only one presentation of a given target direction, even within blocks of trials where the target may, unpredictably, be defined by a different feature (color, in this case), showing that dimensional-weighting accounts cannot, on their own, account for motion direction priming patterns. Finally, we show by randomizing the set size between trials that priming of motion direction can decrease search rates in visual search.
\end{abstract}

When we view a given visual scene, our attention is typically drawn toward items in the visual field that share features with what we have recently attended to (see, e.g., Kristjánsson, 2006a, for a review). Studies of the influence of what has previously occurred on visual search performance have, in many ways, proved themselves to be an effective way of investigating how we allocate attention across the visual field, showing that a visual search for a particular target is faster if the same target appears as appeared on a previous trial (e.g., Becker, 2008; Goolsby \& Suzuki, 2001; Kristjánsson, Wang, \& Nakayama, 2002; Lamy, Antebi, Aviani, \& Carmel, 2008; Maljkovic \& Nakayama, 1994; Olivers \& Meeter, 2006; Treisman, 1992; Wang, Kristjánsson, \& Nakayama, 2005), if the current target shares overlapping features with a preceding target (Kristjánsson, 2006b), or if it appears in the same position as on the last trial (Geng et al., 2006; Geyer, Müller, \& Krummenacher, 2007; Kristjánsson, Vuilleumier, Malhotra, Husain, \& Driver, 2005; Maljkovic \& Nakayama, 1996). Such priming patterns cannot be explained by response repetition or changes in decision criteria (Maljkovic \& Nakayama, 1994; Sigurdardottir, Kristjánsson, \& Driver, 2008), and have shown themselves to be potent enough to account for large portions of effects attributed to explicit top-down guidance in theories of visual attention (Kristjánsson et al., 2002; Wang et al., 2005; Wolfe, Butcher, Lee, \& Hyle, 2003).
Such effects are not, however, confined to target properties, but seem to involve properties of the context that the target appears in, as well (see Geyer, Müller, \& Krummenacher, 2006; Kristjánsson \& Driver, 2008; Kristjánsson et al., 2002; Saevarsson, Jóelsdóttir, Hjaltason, \& Kristjánsson, 2008; Wang et al., 2005) in that the repetition of distractor sets speeds performance independently of target repetition (see, e.g., Kristjánsson \& Driver, 2005, 2008; Lamy et al., 2008). Geyer et al. (2006) have argued that such priming from repeated distractor sets can, in some cases, even overshadow priming from target repetition.

\section{Processing of Visual Motion As a Function of Viewing History}

In the present study, we set out to investigate effects of repeating the direction of motion of a target in a visual search task. Visual search for a target defined by luminancedefined motion (or first-order motion) ${ }^{1}$ in the odd direction has been investigated before, and the findings have generally indicated that motion can be selectively attended to, similarly to other "basic features" to which our visual system seems particularly sensitive, such as color or form (see, e.g., Egeth, Virzi, \& Garbart, 1984; Treisman, 1988; Treisman \& Gelade, 1980; Wolfe, Cave, \& Franzel, 1989). McLeod, Driver, and Crisp (1988) found that detection of a moving $X$ appearing within an array of moving Os and static Xs can be performed in parallel. Dick, Ullman, and

Á. Kristjánsson, ak@hi.is 
Sagi (1987) found similar results for squares moving on a screen (but not for apparent motion over longer distances). Nakayama and Silverman (1986) have found converging results for visual search for motion-defined targets. No studies have, however, addressed priming of visual search for motion-defined stimuli in detail.

Considerable evidence is available showing that our perception of visual motion changes, the longer we view a given motion display. For example, our sensitivity to speed changes can be modulated if we adapt to a moving stimulus for some time (see Clifford \& Langley, 1996; Clifford \& Wenderoth, 1999; Kristjánsson, 2001; see also Clifford, 2002; Kohn, 2007, for reviews). Following prolonged viewing of a drifting pattern, observers tend to report that the apparent speed of the pattern decreases (Clifford \& Wenderoth, 1999; Gibson, 1937; Goldstein, 1957; Kristjánsson, 2001; Ledgeway \& Smith, 1997; Thompson, $1981)$. When the pattern stops moving, it appears to drift in the direction opposite to the previous direction of motion (known as the motion aftereffect; see Anstis, Verstraten, \& Mather, 1998, for a review). And importantly for the present purposes, Chaudhuri (1990) showed that the motion aftereffect is affected by attention, being reduced by a secondary discrimination task.

Perception of motion can thus clearly be modulated by what has occurred previously. In another example, Kanai and Verstraten (2005) found that brief adaptation to unambiguous visual motion results in biases in the perception of a subsequently presented directionally ambiguous motion display, causing either a priming effect, in that the ambiguous motion is seen as drifting in the same direction (if the perceived direction is tested for briefly afterward), or a rapid motion aftereffect (perceived as drifting in the opposite direction), if perceived direction is tested for somewhat later.

Repetition priming of motion direction in visual search has, on the other hand, not been investigated in detail, but some previous studies have indicated that repetition of motion direction can have a facilitatory influence on subsequent visual search performance (see Campana, Cowey, $\&$ Walsh, 2002). They did not test the effects of distractor repetition specifically, however, as is done here. Campana, Pavan, and Casco (2008) then found that priming for motion direction occurs not only with first-order but also with second-order motion. There is some reason to believe that motion does not result in effects in visual search similar to those of some other features (see Franconeri \& Simons, 2003), that motion is more likely to capture attention than are other features. In light of the above, the aim of the present experiments was to obtain a detailed picture of the characteristics of motion priming in visual search.

\section{Can Priming Effects Be Accounted for by Dimension Changes Rather Than Feature Changes?}

An important but unresolved issue regarding priming in visual search is whether priming is dimension-based or feature-based. Some studies have indicated that dimensionbased intertrial effects are stronger than effects from rep- etition of target features (Found \& Müller, 1996; Müller, Krummenacher, \& Heller, 2004). Found and Müller tested search performance, where the targets were defined along two different feature dimensions (orientation and color) within a block of trials, allowing for a direct comparison between the effects of dimension changes (e.g., from red to left-tilted) and within feature changes (e.g., from red to green, or from left-tilted to right-tilted). Their conclusion was that the largest part of intertrial decreases in performance levels could be attributed to dimension changes rather than feature changes. According to the dimensionalweighting account (Found \& Müller, 1996; Müller, Heller, \& Ziegler, 1995; Müller et al., 2004), attention is weighted toward the target-defining dimension in a particular visual search task, and the largest repetition priming benefits (or costs to changes) arise for those features that receive the largest weighting - in particular, those that define the target within a trial block.

Olivers and Meeter (2008) assessed this dimensionalweighting account of intertrial effects, finding that feature-value change effects (e.g., from one color to another, or from one orientation to another) were very similar, whether they occurred within a feature block, where the target-defining dimension remained constant, or within a dimension-change block, where the targetdefining dimension varied unpredictably between being orientation or color. Their observers decided whether a target bar was present or absent among gray vertical distractor bars. The target was defined either by differences in color or by differences in orientation from the distractors. Olivers and Meeter (2008) observed that the feature repetition effects were approximately equal, independently of whether the target-defining dimension remained constant within a trial block or whether the targetdefining dimension changed unpredictably between color and orientation within a block of trials. In other words, it did not seem to have an effect upon feature priming effects what the particular dimensional context was, inconsistent with strong versions of the dimensional-weighting account. In Experiment 4, we will investigate this issue with regard to motion priming.

A final issue we will attempt to address in the present experiments is whether priming has effects on the search process itself rather than on early visual processes or late decisional processes. Many have argued that to show that a manipulation of a visual search task has an effect on the search process itself, slopes of set size versus response times (RTs) must be affected (without a corresponding effect on error rates; see, e.g., Wolfe et al., 1989; but see also Bundesen \& Habekost, 2008, for alternative views). To show that repetition affects search rates in the present context, there must be an interaction between RTs and repetition of the critical features. In Kristjánsson et al. (2002) we did not find effects of priming on search slopes, but Becker and Horstmann (2009) have since found such effects on slopes in a related paradigm. In Experiment 5, we varied the number of items in the search array to assess whether there would be any differences in search slopes as a function of priming. 


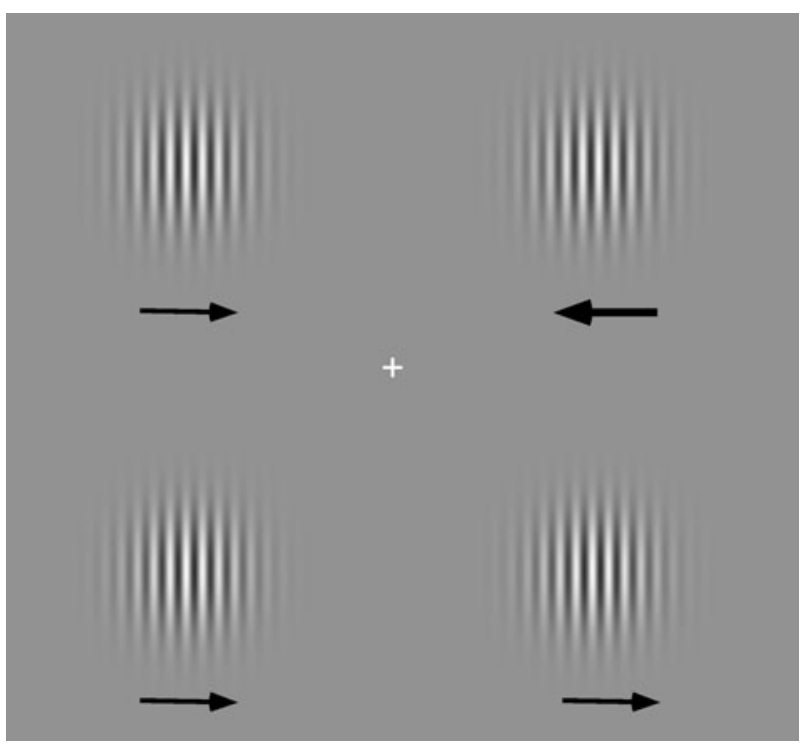

Figure 1. A schematic of a representative search display from the visual search experiments. For each of the four gratings, the sinusoid drifted within the stationary Gaussian envelope (see Method section for more details). In Experiments 1A and 1B, the task was to indicate by keypress whether a target drifting in the odd direction relative to the other three was present on the screen. Otherwise, all four stimuli drifted in the same direction, and the required response was to say that no target was present on the screen. In Experiments 2 and 3, a target was present on all trials, whereas Experiment 4 involved search where the task was to indicate whether a drifting target or odd color target was present or not on the screen (see the respective Method sections for further details on each experiment). The arrows indicate the drift directions on a typical target-present trial (the largest arrow showing the drift direction of the target), but note that the arrows were not actually present on the screen in the experiments. In Experiment 5, a different experimental setup was used (see text).

\section{Overview of the Present Experiments}

The basic task here was visual search for a target moving in a different direction from the other items on the screen (see Figure 1). Using the same basic paradigm as in all our studies, we were able to vary the task relevance and response features in the different experiments and compare and contrast these different aspects of the search within a similar context in an attempt to obtain a relatively thorough picture of the effects of repetition of the direction of luminance-defined motion, both for target and distractor repetition in a visual search task.

Experiments 1-3 were designed to assess the basic characteristics of priming of luminance-defined motion and to determine under which conditions such effects occur (cf. Olivers \& Meeter, 2006). In Experiment 1A, the observers had to indicate whether a target drifting in the odd direction relative to the distractors was present or not on the screen. Testing cases where the target was not present allowed us to investigate the effects of repeating sets of distractors all drifting in the same direction upon search performance, which had not been tested before. As mentioned above, distractor set priming can, in other contexts, have very strong facilitatory effects upon visual search performance (Geyer et al., 2006;
Kristjánsson \& Driver, 2008; Kristjánsson et al., 2002; Lamy et al., 2008; Wang et al., 2005). Experiment 1B was performed to control for some potentially confounding influences on the results from Experiment 1A. In Experiment 2, the observers had to indicate the location on the screen (upper left, upper right, lower left, or lower right quadrant) of the target drifting in the odd direction. In Experiment 3, observers were instructed to indicate the color of a target drifting in the odd direction, relative to the distractors. In Experiment 4, we investigated the significance of the dimensional context within a block of trials for priming of motion - in other words, whether dimension changes of the target-defining feature within a block of trials would eliminate the priming effects, as would be predicted from some accounts of priming in visual search (Found \& Müller, 1996; Müller et al., 1995; Müller et al., 2004; Müller, Riemann, \& Krummenacher, 2003) and was in fact found to be the case under some conditions (Olivers \& Humphreys, 2003; Olivers \& Meeter, 2006; Weidner, Pollmann, Müller, $\&$ von Cramon, 2002). Experiment 5 served two purposes - first, to investigate whether the regular layout used between trials in the foregoing experiments could explain any facilitation effects from repetition of motion direction; and, second, to investigate whether priming has effects upon slopes of RTs versus set size. The latter question, in particular, is important, since it addresses whether priming affects the search process itself rather than some other process, such as response selection or visibility, involved in the task (see Becker \& Horstmann, 2009; Kunar, Flusberg, Horowitz, \& Wolfe, 2007; Sigurdardottir et al., 2008, for discussion of this issue).

\section{GENERAL METHOD}

In all experiments, observers performed visual search tasks in which we contrasted search for Gabor patches with orthogonal motion signals (differing by $180^{\circ}$ from the distractor motion, or by $90^{\circ}$ or $180^{\circ}$ from the distractor motion direction in Experiment $1 \mathrm{~B}$ ). The observers never knew which direction would define the target. The Gabor patches "drifted" locally to the left or the right, in that their Gaussian envelopes stayed in place; this meant that the whole Gabor patch itself stayed in place but the sinusoidal grating within the Gaussian window was phase shifted to the right or left (or left, right, up, or down, in Experiment 1B) at a rate of 5\% 5 sec. This resulted in "local" drift within the Gaussian envelope. The spatial frequency of the Gabors was $1.6^{\circ}$ arc and the size of the Gaussian envelopes was $2.8^{\circ}$ arc at a viewing distance of $60 \mathrm{~cm}$. The brightness of the Gabor patches ${ }^{2}$ varied from 3.2 to $24.5 \mathrm{~cd} / \mathrm{m}^{2}$ and they were always oriented vertically in the first four experiments (as shown in Figure 1), whereas in Experiment 5 their orientation was more heterogeneous. The stimuli were presented on a background with a brightness value at the midpoint of the grayscale used for the Gabors. The target always appeared in a randomly determined location (out of the four possible locations, but note the exception in Experiment 5).

The stimuli were presented on an imaginary circle with a radius of $7.7^{\circ}$ arc away from the screen center where a white fixation cross $\left(30.5 \mathrm{~cd} / \mathrm{m}^{2}\right)$ was at dead center (see Figure 1). Auditory feedback was given after each trial, depending on whether the response was correct (in which case, a $65-\mathrm{Hz}$ sinusoidal tone was played for $5 \mathrm{msec}$ ) or incorrect (in which case, a $55-\mathrm{Hz}$ sinusoidal tone was played for $5 \mathrm{msec}$ following the response). 


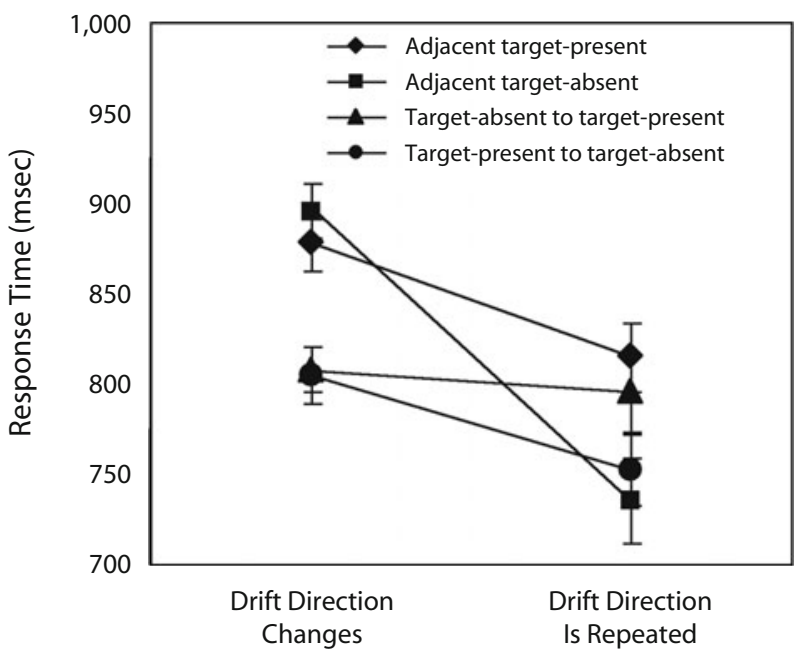

Figure 2. Results of Experiment 1A. The response times are shown as a function of whether the same search is repeated or whether the search type changes - that is, whether the direction of the distractors and the target changes between trials or not (shown for target-present trials preceded by a target-present trial; target-absent trials preceded by a target-absent trial; targetpresent trials preceded by a target-absent trial; and target-absent trials preceded by a target-present trial). Error bars show the standard error of the mean for each data point.

\section{Equipment}

The stimuli were presented on an $85-\mathrm{Hz}$ CRT display with a screen resolution of $768 \times 1,024$ pixels, driven by a standard video card on a G4 PowerMac. Custom software for stimulus generation, presentation, and data collection was prepared in the C programming language utilizing the Vision Shell function library (Comtois, 2003). Stimulus presentation, timing control, and RT measurements were controlled by a Macintosh G4 computer. Any deviations in each individual experiment from the general methodology described here will be explained in their respective Method sections.

\section{EXPERIMENT 1A}

\section{Method}

In Experiment 1A, the observers had to indicate whether or not a target drifting in an odd direction relative to the other stimuli was present on the screen. Each trial started with the presentation of a fixation cross on a mid-gray background. The observers had to decide whether a target of odd drift direction (either drifting to the right among distractors drifting to the left, or drifting to the left among distractors drifting to the right, either of which was a target-present trial) was present on the screen or not. A target was present on $50 \%$ of the trials. If no target was present, all the Gabor patches drifted in the same direction (a targetabsent trial). Observers responded by keypress and were told to try to respond as quickly as possible, without sacrificing accuracy. Each observer participated in 300 trials following 30 practice trials. Nine observers ( 5 male) participated, ranging in age from 18 to 32 years. They all had normal or corrected-to-normal vision. A trial started with the presentation of a fixation cross at the center of the screen. Twelve hundred to $1,700 \mathrm{msec}$ afterward (determined randomly for each trial), the four drifting gratings were presented until the observer responded, after which another trial followed, 1,200 to $1,700 \mathrm{msec}$ later (during which the fixation cross was present on the screen).

\section{Results}

Figure 2 shows the mean RTs from Experiment 1A as a function of repetition type between adjacent trials. Error rates are shown in Table 1. The critical manipulation is whether or not the same search type is repeated (in essence, whether the distractors drifted to the left or the right, with a target present or not). Shown are the mean effects of repetition for the 9 observers for adjacent target-present trials, adjacent target-absent trials, target-present trials preceded by target-absent trials, and target-absent trials preceded by target-present trials. The results clearly indicate that search is speeded if the same search type is repeated as on the previous trial. Repeating the drift direction of the target speeds performance on a subsequent target-present trial. Repeating the drift direction of the distractor sets between adjacent target-absent trials similarly facilitates performance. Also, if the same distractor types appear on a target-absent trial as on a previous target-present trial, performance is speeded. The only repetition pattern that did not result in a significant speeding effect (see below) was when distractors were repeated on a target-present trial preceded by a target-absent trial, although there was surely a trend toward such an effect.

Paired $t$ tests indicated a significant difference for repetition of motion direction of the target for adjacent targetpresent trials $[t(8)=6.53, p<.01],{ }^{3}$ for adjacent targetabsent trials $[t(8)=12.72, p<.01]$, and for repeating distractors from a target-present trial upon a subsequent target-absent trial $[t(8)=3.97, p<.01]$, but the effect of repetition was not quite significant for target-present trials preceded by a target-absent trial $[t(8)=1.47, p>.05]$, although the pattern surely indicated that such an effect is, most likely, there. Similar $t$ tests on the error rates showed that they did not significantly change for any repetition type (all $F \mathrm{~s}<1$ ).

The results from Experiment 1A indicate that repetition of motion direction has a strong effect on attention deployments in visual search for the odd motion direction. The results show a strong effect not only of target repetition but also of distractor repetition, as seen before in different contexts for different stimulus types by Geyer et al. (2006), Kristjánsson and Driver (2005, 2008), Kristjánsson et al. (2002), and Wang et al. (2005). In fact, judging by Figure 2, the largest effect is in fact seen for adjacent target-absent trials, and the $t$ tests reported above support the contention that this effect was the most reliable (but see Experiment 5, where some caveats to this conclusion are discussed and addressed experimentally).

Note, however, that distractor identity and target identity did not vary independently of each other in Experiment $1 \mathrm{~A}$, so repetition effects due to target repetition and distractor repetition cannot be easily disentangled within this design. It is theoretically possible that distractor repetition actually accounts for most, if not all, of the priming

Table 1

Error Rates From Experiment 1A As a Function of Repetition Type

\begin{tabular}{lcccc}
\hline & TP to TP & TA to TA & TA to TP & TP to TA \\
\hline No repetition & 3.1 & 3.9 & 4.5 & 3.3 \\
Repetition & 3.5 & 4.1 & 4.2 & 2.9 \\
\hline
\end{tabular}

Note-TA, target-absent trial; TP, target-present trial. 


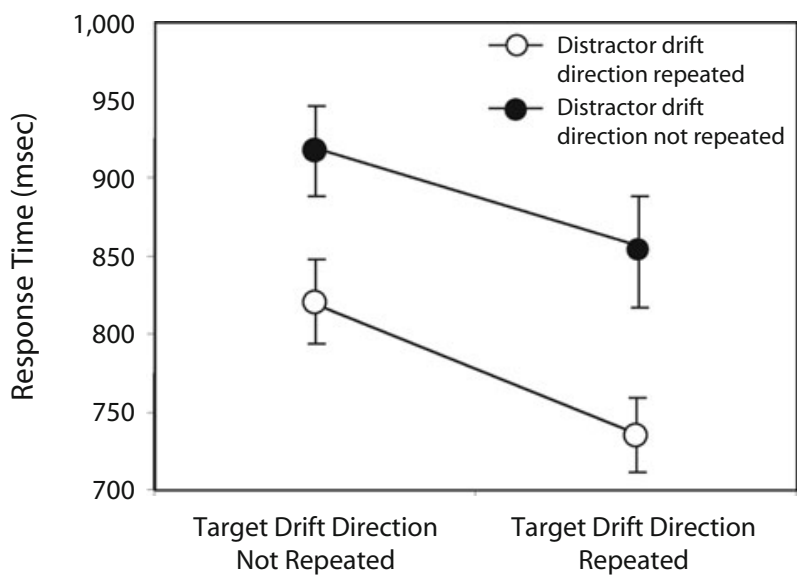

Figure 3. Results of the target-present trials from Experiment $1 \mathrm{~B}$ as a function of whether or not the target drift directions (filled disks) and distractor drift directions (white disks) were repeated. Excluded are trials where distractor identity is the same as the target identity on the last trial (see the text for details). Error bars show the standard error of the mean.

effects seen (see Geyer et al., 2006, for examples), since each time the target direction is repeated, so is distractor direction. But since the only repetition pattern that did not result in a statistically significant speeding effect was when distractors were repeated on a target-present trial preceded by a target-absent trial, this argues against the view that all the priming effects can be accounted for with distractor repetition, which cannot be considered a strong inference. Experiment 1B was therefore performed in an attempt to disentangle the effects of repeating target motion direction, versus repeating distractor motion direction.

\section{EXPERIMENT 1B}

\section{Method}

In Experiment 1B, the target and distractor orientation and drift rates varied independently in an attempt to disentangle effects of repeating the target and repeating the distractors, since in Experiment $1 \mathrm{~A}$, each target direction was repeated, as was distractor drift direction. Instead of two possible drift directions, as in Experiment $1 \mathrm{~A}$, there were now four possible drift directions for the targets and distractors (up, down, left, or right). As in Experiment 1A, the observers were instructed to indicate whether a target drifting in an odd direction relative to the other Gabors was present or not on the screen. Seven observers ( 5 female) participated in 300 trials each in blocks of 100 trials with 30 practice trials run beforehand. All had normal or corrected-to-normal vision. In other respects, methods were similar to those described in the General Method section and for Experiment 1A. Since the main question of interest was disentangling the effects of target and distractor priming, only results for the target-present trials are reported.

\section{Results}

Figure 3 presents the results from Experiment 1B. Contrasted is performance on trials where the target drift direction is repeated or not, and where distractor identity is repeated or not, excluding trials where distractor identity is the same as the target identity on the last trial, or vice versa, since if the distractor identity becomes the target identity on the next trial, this may lead to the potential confounding effects from Experiment 1A that the experiment was designed to avoid.

In short, the results from Experiment 1B show that the repetition effects for the target-present trials in Experiment $1 \mathrm{~A}$ cannot all be attributed to distractor repetition, since the benefits of repeating the target drift direction are considerable in Experiment 1B (even when the distractor drift direction changes). They are almost as strong as the effect of repeating distractors (measured as differences in RTs). The largest repetition benefit is, however, seen when both distractors and target are repeated (as seen before by Kristjánsson \& Driver, 2005, 2008, and Lamy et al., 2008). A $2 \times 2$ repeated measures ANOVA confirmed this, showing that the effect of repetition of target drift direction upon RTs was significant $[F(1,6)=17.71, p<$ $.01]$, as was the effect of repeating the drift direction of the distractors $[F(1,6)=23.2, p<.005]$. There was, however, no interaction between target and distractor repetition $[F(1,6)=1.06, p=.34]$, again confirming the independence of the effects of target versus distractor repetition. The error rates are shown in Table 2 and do not give any indication of a speed-accuracy trade-off as a function of motion direction repetition (all $F_{\mathrm{S}}<1.3$ ).

In sum, the combined results of Experiments 1A and 1B clearly show that the facilitatory effects of repetition of motion direction on both target and distractor processing are quite strong, speeding attention shifts to the target in addition to speeding the decision that no target is present, presumably through facilitated processing of the distractor identity, so that they can more quickly be rejected as nontargets (see Geyer et al., 2006; Kristjansson \& Driver, 2008; Lamy et al., 2008; Wang et al., 2005).

\section{EXPERIMENT 2}

\section{Method}

Experiment 2 was performed to investigate whether judgments of the location of a target drifting in the odd direction would be speeded with repeated motion direction. Although the results from the previous experiments indicate that priming leads to facilitated detection of a target, it does not necessarily follow that processing of the location of the target is also facilitated on subsequent trials. The task of the observers in Experiment 2 was to indicate the location of the Gabor patch drifting in the orthogonal direction (left or right) to the three distractors (a target was this time present on all trials). Each of the 8 observers ( 5 male) participated in 30 practice trials followed by 300 experimental trials run in two blocks of 150 trials. All the observers had normal or corrected-to-normal vision. They were required to indicate by pressing one of four keys where in the visual field a target drifting in the odd direction was located. The response alternatives were to press " 1 " for the odd target in the lower left visual field, " 3 " for a target at the lower right, "7" for a target at the upper left, and "9"

Table 2

Error Rates From Experiment 1B

As a Function of Repetition Type

\begin{tabular}{lcc}
\hline & Target Not Repeated & Target Repeated \\
\hline Distractors not repeated & 3.5 & 4.6 \\
Distractors repeated & 5.7 & 3.7 \\
\hline
\end{tabular}




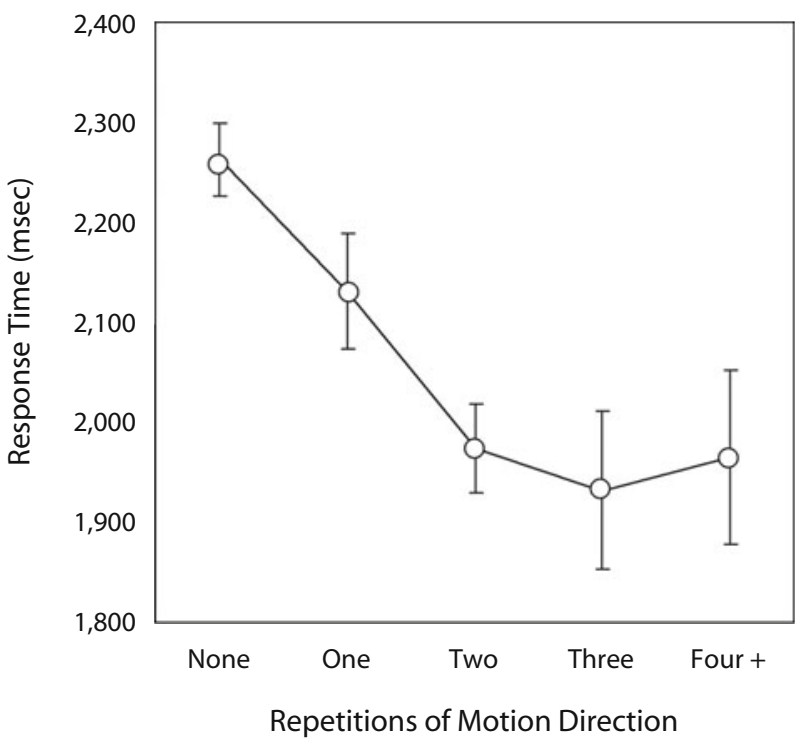

Figure 4. Results from Experiment 2. The figure shows the mean response times from Experiment 2 as a function of how often the direction of motion on the target is repeated. Error bars show the standard error of the mean.

for the upper right. In other respects, methods were similar to those described in the General Method section and for Experiment 1A.

\section{Results}

Figure 4 shows performance in Experiment 2 as a function of the repetition of motion direction, for zero, one, two, three, and four or more repetitions of target motion direction. The error rates as a function of repetition are shown in Table 3, and again they give no indication of any speed-accuracy trade-off $[F(4,32)=0.87, p=.49]$. The figure indicates that as motion direction is repeated, location judgments of the target (which was always present) are increasingly facilitated. A repeated measures ANOVA confirmed that this repetition effect was highly significant $[F(4,32)=16.78, p<.001]$.

The results from Experiment 2 indicate that the repetition speeds the location judgment by $92.5 \mathrm{msec}$, on average, for each repetition. This result further strengthens our conclusions from Experiments 1A and 1B, that repeating motion direction has a strong effect on how we allocate attention across the visual field, and that the influence is on location judgments of where the target appears, not just whether or not it was present.

\section{EXPERIMENT 3}

\section{Method}

The previous experiments in this article have shown the importance of repetition of target motion for judgments of a target's presence (or absence) and for judgments of a target's location. Experiment 3 was performed to test the effects of repeating target motion in a visual search task upon judgments of the target's color, a feature unrelated to the search. The observers were required to indicate by keypress the color scale (redscale or greenscale) of the target Gabor patch drifting in the odd direction. As in Experiment 2, a target was now present on all trials.
Procedure. As before, each trial started with the presentation of a fixation cross on the mid-gray background. Twelve hundred to $1,700 \mathrm{msec}$ afterward (determined randomly for each trial), the four drifting Gabor patches were presented until the subject responded. The observers had to indicate whether the target drifting in the odd direction was a "redscale" Gabor patch, varying in brightness from dark red $\left(4.8 \mathrm{~cd} / \mathrm{m}^{2}\right)$ to light red $\left(33.7 \mathrm{~cd} / \mathrm{m}^{2}\right)$, or a "greenscale" Gabor patch, varying in brightness from dark green $\left(5.8 \mathrm{~cd} / \mathrm{m}^{2}\right)$ to light green $\left(26.3 \mathrm{~cd} / \mathrm{m}^{2}\right)$. Two of the Gabor patches were greenscale and two redscale, determined randomly for each trial. At the edges of the Gabor patches, the brightness values were at the midpoint value of the green scale or red scale. Eight observers (6 female) participated. Each observer participated in 300 trials preceded by 30 practice trials. In other respects, methods were similar to those described in the General Method section and for Experiment 1A.

\section{Results}

Direction is the target-defining feature in Experiment 3, but not the response-defining feature; color is. Figure 5 shows the mean RTs, as a function of repetition of motion direction on the target defined by the odd motion direction from Experiment 3. The error rates are shown in Table 4, and once again give no indication of a speed-accuracy trade-off as a function of motion direction repetition $[F(4,28)=1.45, p=.24]$. There was a clear benefit of repeating the motion direction of the single oddball target (in terms of motion). A repeated measures ANOVA confirmed that this beneficial effect of repetition of motion direction was reliable $[F(4,28)=$ $13.12, p<.001]$. This further strengthens our conclusions from previous experiments that motion repetition on the target speeds attention shifts, and, importantly, also indicates that motion direction does not need to be the response-defining feature of the visual search task for such facilitation to occur (see also Kristjánsson, 2009), since the task of the observer was to identify the color scale of the target Gabor patch.

\section{EXPERIMENT 4}

The results of the previous experiments indicate that motion direction does not need to be the response-defining feature for priming from repeated motion direction to occur. One may ask, however, whether such priming is dependent on motion direction being the target-defining feature throughout a whole block of trials for priming to occur. Whereas some research indicates that motion direction need not necessarily be task relevant for motion direction priming to occur (Kristjánsson, 2009), this question has not been addressed explicitly in previous research. As was discussed at the beginning of this article, there has been debate over whether effects of dimensional changes can overshadow or even account for priming in visual

Table 3

Error Rates From Experiment 2 As a Function of Repetition of Motion Direction on the Target

\begin{tabular}{ccccc}
\hline Zero & One & Two & Three & Four + \\
\hline 2.9 & 3.9 & 4.2 & 3.8 & 3.7 \\
\hline
\end{tabular}

Note-No repetition to four or more repetitions. 


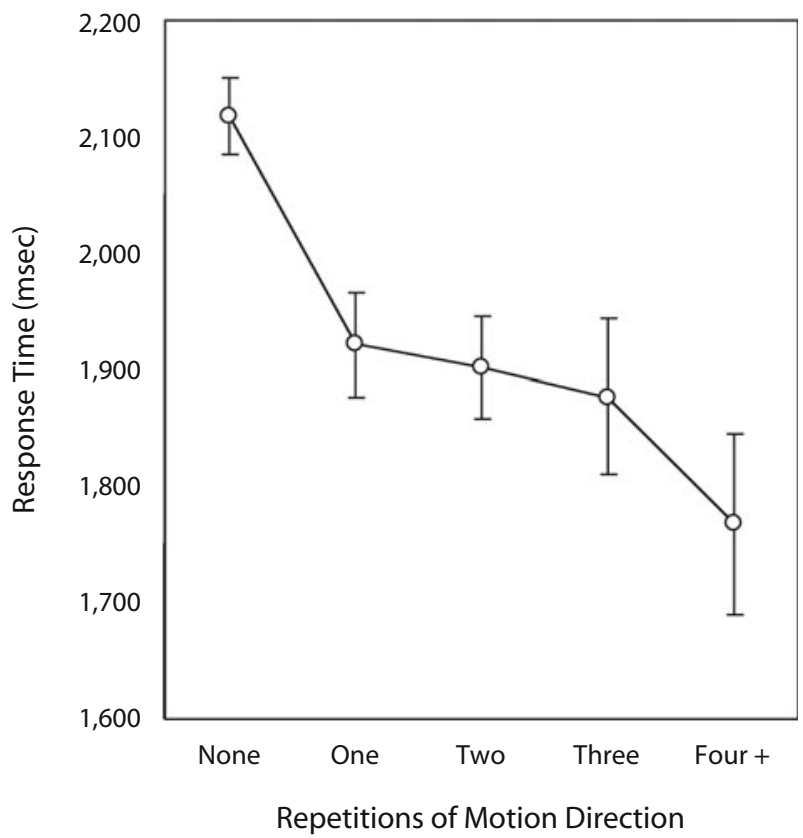

Figure 5. Results from Experiment 3. Shown are the mean response times as the motion direction on the target is repeated a given number of times. Error bars show the standard error of the mean.

search. Found and Müller (1996) had their observers search for a target defined by either color or orientation and found that intertrial facilitation was largely dimension specific rather than feature specific. They argued that the size of priming effects should reflect the task relevance of a given feature dimension. As Olivers and Meeter (2008) have suggested, it may be a reasonable strategy for the visual system to place more weight on dimension changes than on changes in features only. Olivers and Meeter (2008) found, however, that the effects of feature changes were approximately the same for blocks where there was dimensional and feature change as when there were feature changes only; this would not be predicted by a strong version of the dimensional-weighting account, since by such accounts, the priming effects should be strongest by far for the dimension defining the target within a trial block (see Müller et al., 1995).

In Experiment 4, we tested such effects of the dimensional context upon repetition effects for motion direction, using static grayscale Gabor patches as distractors; the task was to indicate whether an odd target (either a grayscale Gabor drifting to the left or the right, or a stationary redscale or greenscale Gabor patch) was present or not. If the dimensional context (whether the target-defining dimension is constant or variable throughout a block) affects priming from repeated features, there should be an effect upon feature repetition priming patterns, whether the target is defined by color or motion direction throughout a block of trials or whether the target-defining dimension changes within blocks of trials. On the other hand, if the target-defining dimension need not be constant throughout a block for priming to occur, we should see priming from feature repetition, even in trial blocks where the target-defining dimension changes, and this should not differ significantly as a function of whether only the feature values (color or motion) change, or whether the target-defining dimension also changes within blocks.

As explained above, Found and Müller (1996) observed that the effects of dimension changes dwarfed the effects of feature repetition. This is in line with the dimensionalweighting account of priming. If the dimension-weighting account is to account for the results from Experiment 4, we should also see such a pattern in our results here, in that the feature change effects should be smaller than the dimension-change effects (as observed by Olivers \& Meeter, 2008), although Olivers and Meeter (2008) nevertheless found strong feature repetition effects within the dimension-change blocks, which is not consistent with a strong version of the dimension-weighting account, for reasons explained above.

\section{Method}

The task of the observers was to indicate whether a target was present or not among static grayscale Gabor patches. The target was defined either by motion direction (a grayscale Gabor patch drifting to the left or right, as in previous experiments) or by color (the target was a "redscale" or "greenscale" stationary Gabor patch; see Method section of Experiment 3 for details). A target was present on $60 \%$ of the trials, in which case three static grayscale Gabors (distractors) were also present, whereas on a target-absent trial, four static grayscale Gabor distractors were presented (the four possible positions were those shown in Figure 1). Six observers (4 female) participated in 600 trials in the dimension-change condition and 300 trials in each of the single-feature conditions, 1,200 trials in total. These blocks were run in counterbalanced order for the different subjects to control for any practice effects. In other respects, methods were similar to those described for the preceding experiments.

\section{Results}

Figure 6A shows the average cost of switches, either between dimensions in the dimension-change blocks or within feature changes (color change or motion direction change on the target) for both the blocks with dimension changes and the single-feature blocks. The error rates as a function of repetition of target features and block type are shown in Table 5.

Although the dimension-change effect is the largest, there are also large effects of feature repetition for both types of trial blocks. This result means that the repetition effects can hardly be described as being "largely dimension based." Clearly there are costs to changing the targetdefining dimension within a block of trials, but at the same time there are also strong effects of within-feature changes for both block types. The most critical question was whether intertrial effects for features would differ for dimension-change blocks and single-feature blocks.

Table 4

Error Rates From Experiment 3 As a Function of Repetition of Motion Direction on the Target

\begin{tabular}{ccccc}
\hline Zero & One & Two & Three & Four+ \\
\hline 5.8 & 4.2 & 3.2 & 3.8 & 2.9 \\
\hline
\end{tabular}

Note-No repetition to four or more repetitions. 
A

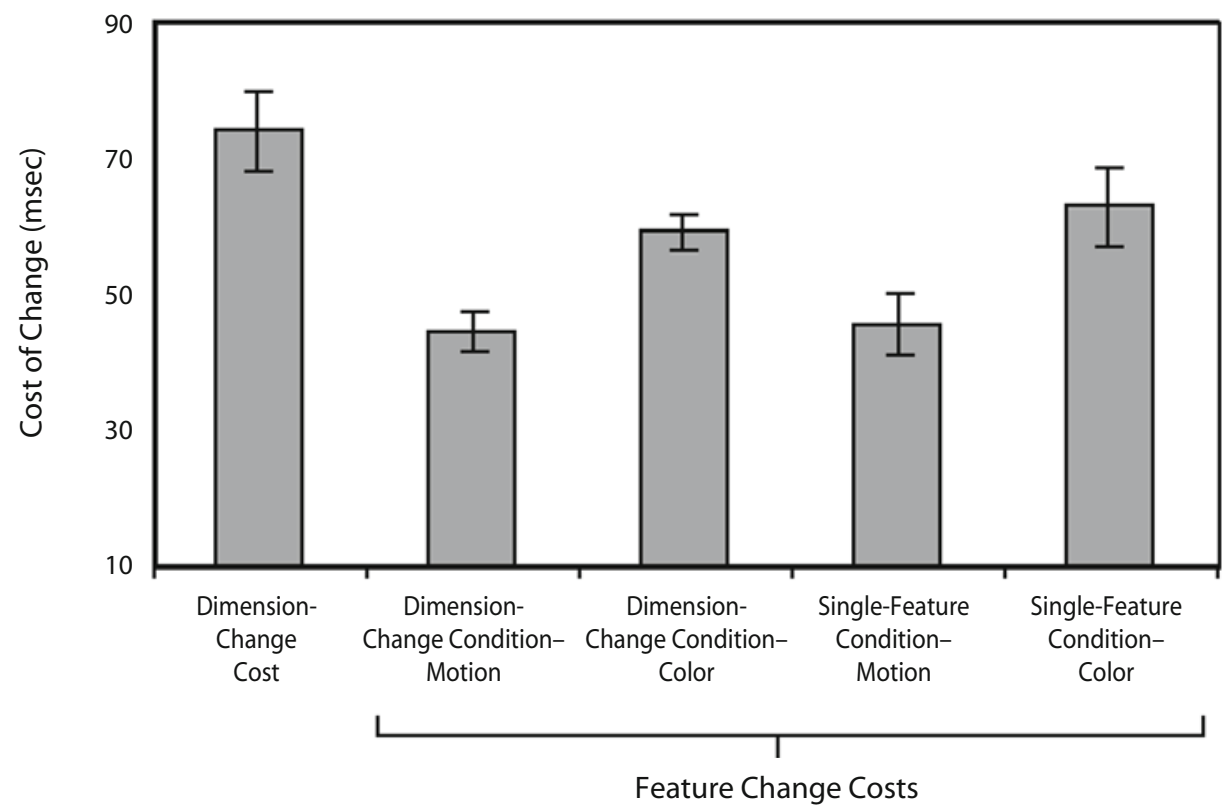

B

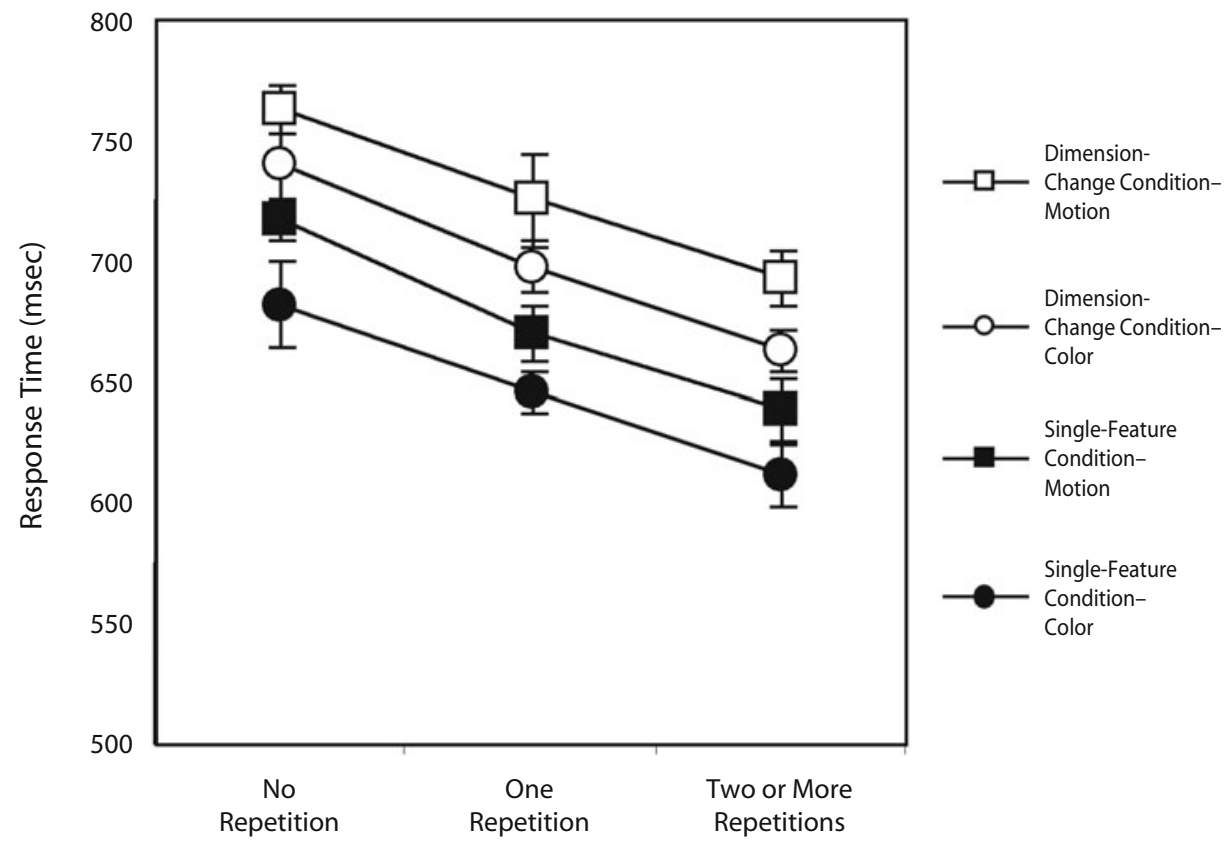

Figure 6. Results from Experiment 4. (A) The cost of switching dimensions, and of switching features under dimension-change blocks and single-feature blocks. (B) The repetition effect as a function of the two different conditions (dimension-change blocks or single-feature blocks), and of repeating the different features (color or motion) a given number of times.

A 2 (dimension-change block vs. single-feature block) $\times$ 2 (motion repetition vs. color repetition) ANOVA was performed to test the reliability of these effects. Importantly, the effect of block type upon the intertrial effects was $n o t$ significant $[F(1,7)=0.317, p=.591]$, but the effect of feature type was significant $[F(1,7)=28.3, p<$ $.001]$, confirming that the effects of color repetition were somewhat larger than the motion repetition effects. The interaction between the two factors was not significant, however $[F(1,7)=0.323, p=.588]$, showing that the 
Table 5

Error Rates From Experiment 5 As a Function of Repetition of Target Features and Block Type

\begin{tabular}{lcccc}
\hline & DC Motion & DC Color & SF Motion & SF Color \\
\hline No repetition & 4.2 & 4.7 & 3.3 & 4.8 \\
One repetition & 4.3 & 4.8 & 3.2 & 2.0 \\
Two repetitions & 4.1 & 5.2 & 2.4 & 3.2 \\
\hline
\end{tabular}

Note-DC, dimension-change block; SF, single-feature block.

effects of dimensional context upon intertrial effects did not differ significantly as a function of which feature defined the target. However, the most important result from Experiment 4 is that the featural repetition effects were comparable for the two conditions, where the targetdefining dimension could change unpredictably between trials and where the target-defining dimension stayed constant within a trial block. A similar ANOVA on the error rates revealed no hints of a speed-accuracy tradeoff (all $\left.F_{\mathrm{s}}<1.56\right)$.

Figure 6B then contrasts the effect on absolute RTs of zero, one, two, or more repetitions of motion direction and color for the dimension-change blocks and the singlefeature blocks. If the effect of feature repetition is differential depending on the dimensional context, we should find an interaction in the effects of repetition. There were, however, no hints of such an interaction. A 2 (block type) $\times$ 2 (feature type) $\times 3$ (zero, one, or two repetitions of a particular feature) repeated measures ANOVA was performed on the RTs (see Figure 6B for mean RTs). There was a significant effect of block type on the RTs $[F(1,7)=86.29$, $p<.001]$ and a significant effect of repetition $[F(2,14)=$ 24.3, $p<.001]$, whereas the effect of feature type was not quite significant $[F(1,7)=3.01, p=.126]$. None of the interactions (two-way or three-way) was significant. The most important interaction term of the ANOVA, for our purposes, was the interaction between block type and repetition. If the effect of featural repetition were differential as a function of dimensional context, there should be an interaction between block type and repetition, since the effect of repetition should be differential dependent on dimensional context, but this was not the case $[F(2,14)=$ $0.054, p=.947]$.

To summarize: The results of Experiment 4 clearly show that priming of motion direction (or color, for that matter) is not dependent on motion direction (or color) being the target-defining dimension throughout a block of trials. There was no difference in the size of the repetition effects as a function of whether the target-defining dimension could change or not within a block of trials. Priming can build up almost instantaneously, even with only one repetition of the critical feature, as seen in Figure 6B. This means that following presentation of a single trial with motion as the target-defining dimension, attention is biased to the motion direction on the last trial, and this occurs even when the target-defining dimension may change unpredictably from one trial to the next. This result is clearly not consistent with a strong version of the dimension-weighting account of priming (as similarly argued in a different context by Olivers \& Meeter, 2008). It seems that explanations in terms of the dimensional-weighting account of priming cannot account for the whole pattern of results; a feature facilitation explanation would seem to be an equally likely explanation. The most parsimonious account for the results is, however, one in which both feature repetition and dimensional context are assumed to affect the RTs.

\section{EXPERIMENT 5}

Experiment 5 addresses two potentially complicating factors regarding the foregoing experiments. First, in the preceding experiment, search is fastest if target direction is repeated in the same location - that is, if the global "gestalt" of the display is repeated, in essence if the exact same display is repeated (see Wolfe, Friedman-Hill, \& Chun, 1991). This would mean that we are not strictly observing facilitation of features (in this case, motion), but facilitation of the processing of the whole display (both target and distractors) instead. The results of some of the previous experiments are, in fact, consistent with this possibility. ${ }^{4}$ Another reason to consider this a distinct possibility is that Kristjánsson, Vuilleumier, Schwartz, Macaluso, and Driver (2007), in a functional magnetic resonance imaging study of feature and position priming, found activity modulations in the fusiform gyrus of the temporal lobe specific to when both the target and distractors were of the same color and in the same locations as on the preceding trial, meaning that the whole display was identical to that on the previous trial; in other words, these were activity modulations specific to whether the whole display was repeated or not.

In some of the previous experiments, observers' judgment may simply have been facilitated by the repetition of every motion direction in the same locations as on the previous trial. In fact, inspection of the RTs from Experiment $1 \mathrm{~A}$ reveals that they are consistent with this possibility. It is thus possible that the priming seen may simply reflect that decisions are facilitated when the same display is repeated. This possibility is consistent with recent demonstrations of priming from distractor sets (Kristjánsson \& Driver, 2008; Lamy et al., 2008) and the importance of regular layout for position priming (Geyer et al., 2007). It must be noted, however, that the results from Experiment 2 argue against such a "global gestalt" argument since, by that argument, no priming effects should be seen when a target must be located; observers should not know where the target appeared, if all that is primed is whether everything is the same as on the previous trial. The results from Experiment 1B also argue against such accounts, since there the distractor direction was not necessarily repeated, but priming effects were about equal to those seen in Experiment 1A. Neither of these counterarguments would seem entirely conclusive, however, calling for further investigation of this issue.

In Experiment 5, we attempted to address this potentially complicating issue with a set-size manipulation, using set sizes of four, six, and eight display items presented in random order within trial blocks. In addition, the Gabor patches took on a random color (half of them were redscale 
A

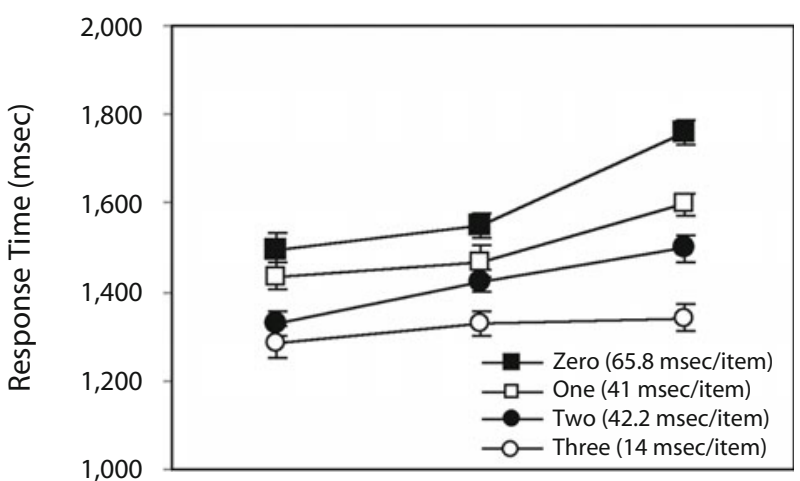

B

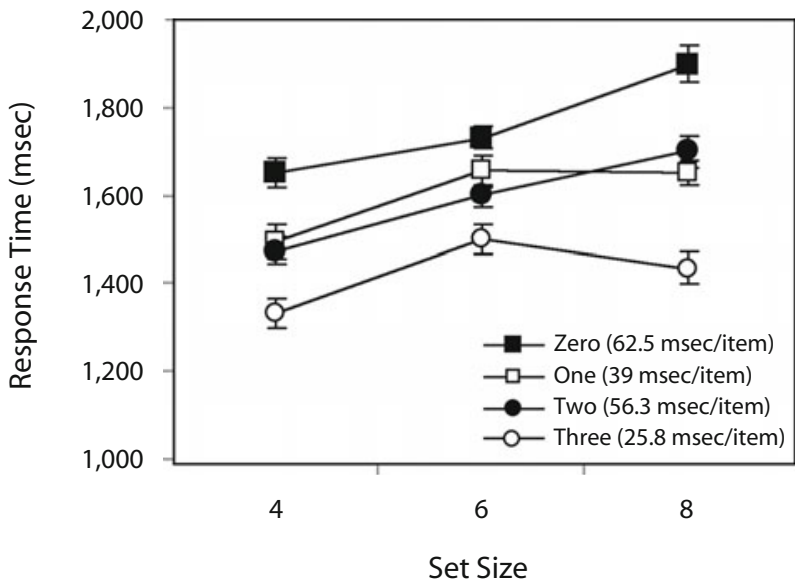

Figure 7. Results of Experiment 5 for (A) target-present trials and (B) target-absent trials. Separate symbols denote how often a given target and distractor direction had been repeated. The figure also shows the slopes of set size versus response times for each repetition. Error bars show the standard error of the mean.

and half greenscale on each trial), and their positions were jittered randomly by $0.5^{\circ}$ (in eight possible directions from the respective locations on the imaginary circle that they appeared on). Furthermore, they were assigned random orientations, either $+5^{\circ}$ or $-5^{\circ}$ away from vertical (half of the search items of each orientation on any given trial). All in all, this results in considerable irregularity of the search array, which means that the possibility of the same display's appearing on two consecutive trials is minimal $(1 / 1,536$, to be precise). If we see motion priming in adjacent trials between different set sizes (and given all the unpredictability regarding the display), we should be able to rule out "gestalt priming" accounts as the sole explanation for the priming effects seen in the previous experiments.

In addition to the potentially complicating issue of whether the priming effects seen in previous experiments reflect priming of the whole display, this experiment can also address whether priming affects the search process itself, rather than perceptual processes preceding it or decisional ones following it.
In a study related to this issue, Kunar et al. (2007) found that search rates did not decrease as contextual cuing (another type of history effect; see Chun \& Jiang, 1998) developed; and they argued that contextual cuing might reflect facilitation of response selection rather than effects upon the search process itself. Kristjánsson et al. (2002) found, for priming of orientation in a visual search task, that the priming effects did not change the slopes of set size versus RTs. Recently, however, priming has been found to affect search slopes (see Becker \& Horstmann, 2009 ) in a modified version of the task used by Kristjánsson et al. (2002), arguing against response facilitation accounts of priming (see also Sigurdardottir et al., 2008). Note also that the location and color judgment tasks used in Experiments 2 and 3 argue against this interpretation, as mentioned above, since the response requirements are orthogonal to the task; this cannot be considered "smoking-gun" evidence, however. The observers had to decide whether a target Gabor drifting in the odd direction relative to all the other Gabors was present on the screen or not. The methods used in Experiment 5 were otherwise similar to those described for the previous experiments, except for the changes detailed above.

\section{Results}

The results of Experiment 5 are shown in Figure 7, separately for target-present trials (panel A) and target-absent trials (panel B) as a function of repetition of target and distractor motion along with the set size. The error rates are shown in Table 6 and do not (as in previous experiments) give any indication that speed-accuracy trade-offs occurred with repetition (all $F_{\mathrm{s}}<0.9$ ).

The results of Experiment 5 address two important issues. First, the potential confound of previous experiments - that what the observers were remembering (or, more precisely, were primed on) across trials was a memory trace of the global "gestalt" of the display rather than motion direction of the individual items - is eliminated, since the likelihood that the exact display as on the last trial would be repeated was so low $(1 / 1,536)$ that it is impossible that repetition of the whole display can account for the repetition priming pattern observed. There are strong priming effects, both from repeating target direction across adjacent target-present trials as well as from distractors (see below). Importantly, there was also strong

Table 6

Error Rates From Experiment 5 As a Function of Set Size, How Often a Given Target and Distractor Direction Were Repeated, and Whether the Target Was Present or Absent

\begin{tabular}{lrrrr}
\hline & Zero & One & Two & Three \\
\hline Set size 4 & 3.9 & Targets & \\
Set size 6 & 2.4 & 3.4 & 3.3 & 2.5 \\
Set size 8 & 4.0 & 4.5 & 5.7 & 3.1 \\
& & Distractors & & \\
Set size 4 & 4.3 & 4.6 & 4.1 & 3.3 \\
Set size 6 & 2.6 & 3.8 & 5.2 & 3.1 \\
Set size 8 & 6.4 & 2.2 & 2.7 & 5.1 \\
\hline
\end{tabular}


Table 7

Effects (in Milliseconds) of Trials of Different Set Sizes (Trials $N-1$ ) on Subsequent Trials (Trials $N$ ) of the Three Different Set Sizes, Shown Separately for Target-Present and Target-Absent Trials

\begin{tabular}{cccc}
\hline & \multicolumn{3}{c}{ Trial $N-1$} \\
\cline { 2 - 4 } Trial $N$ & 4 & 6 & 8 \\
\hline \multicolumn{4}{c}{ Target Present } \\
4 & 45 & 56 & 69 \\
6 & 49 & 67 & 90 \\
8 & 76 & 95 & 156 \\
& Target Absent & \\
4 & 110 & 65 & 79 \\
6 & 104 & 121 & 103 \\
8 & 96 & 88 & 133 \\
\hline
\end{tabular}

priming across different set sizes (see Table 7), which makes it even more unlikely that global gestalt repetition accounts can explain for the results. As an example, a trial with no target and a set size of four leads to priming effects upon subsequent trials of set size six (average priming effect, $65 \mathrm{msec}$ ) and set size eight (average priming effect, $79 \mathrm{msec}$ ), in addition to its effect upon a subsequent trial with set size four.

Another important issue that this experiment addresses is that of the effects of priming upon slopes of set size versus RTs. If we find that slopes of set size versus RTs are affected by repetition of target or distractors, what is affected is the search itself, rather than processes preceding or following it (as these processes are traditionally defined; see Wolfe et al., 1989, for a discussion, and Sternberg, 1969, for the general logic). Note, however, that some have strongly argued against such notions of serial processing stages of search (e.g., Bundesen, 1990; Bundesen \& Habekost, 2008; Shibuya \& Bundesen, 1988).

In short, we find strong effects of priming on search slopes, for both the target-present and target-absent trials. A 3 (set size) $\times 4$ (repetition) $\times 2$ (presence vs. absence) ANOVA showed that the effect of set size was significant $[F(2,14)=6.64, p<.01]$, as were the effects of repetition $[F(3,21)=10.01, p<.001]$ and whether a target was present or not $[F(1,7)=4.16, p<.05]$. The interaction between effects of repetition and the effects of set size was significant $[F(6,42)=4.90, p<.001]$, indicating that repetition priming of motion affected the slopes of set size versus RTs. The other three interaction terms were not significant, however, which shows that slopes were influenced by priming on both target-present and target-absent trials. This result is consistent with arguments that priming affects sensitivity in the search itself, since search rates (RTs as a function of set size) decrease as motion direction of the target is repeated. We further investigated the priming patterns by breaking down the effect of repetition of direction for a particular set size on a subsequent trial of each set size; these effects are shown in Table 7. Clearly, the effect of repeating direction on the target and distractors is not dependent on repeating the same set size, which strengthens the conclusion that the repetition priming effects we have seen in this experiment reflect repetition of features.

\section{GENERAL DISCUSSION}

Our visual systems tend to use the past to predict the future, and for this reason usually prefer consistency over uncertainty. Experimental results have repeatedly shown how our attention is drawn toward what we have recently viewed and what has been important to us in the recent past for one reason or another-for example, a target in a visual search task (see the discussion at the beginning of this article or Kristjánsson, 2008, for a review). It has been argued that priming effects of this sort reflect our possession of a primitive memory system facilitating attention deployments, allowing us to quickly reorient to recently viewed stimuli that are of behavioral importance to us (Kristjánsson \& Nakayama, 2003; Magnussen \& Greenlee, 1999; Maljkovic \& Nakayama, 1994, 2000).

The results from the six experiments reported here strongly indicate that motion direction of targets and distractors in visual search strongly influences where we subsequently allocate our attention. Specifically, these results show how important it can be whether the relevant motion direction is repeated or not between trials. This repetition effect was shown in a number of different contexts: Repetition of target motion direction facilitates target detection when a decision must be made as to whether such a target is present or not on the screen (Experiments 1A and 1B); location judgments of the target drifting in the odd direction are facilitated with repetition (Experiment 2), as are color judgments of the target drifting in the odd direction (Experiment 3). Furthermore, the results from Experiments $1 \mathrm{~A}$ and $1 \mathrm{~B}$ show, for the first time, that repetition of distractor motion also facilitates the search, independently of any target priming. This is consistent with claims of the importance of the priming of context, or distractor sets, previously observed by Geyer et al. (2006), Kristjánsson and Driver (2005, 2008), Kristjánsson et al. (2002), Lamy et al. (2008), and Wang et al. (2005). Experiment 4 then shows that, for priming to take place, motion direction need not be the target-defining dimension throughout a whole block of trials, and that even a single repetition of motion direction within a block of trials in which motion direction or color is the target-defining dimension is enough to result in priming effects (see Figure 6B). Finally, the results of Experiment 5 show that motion priming affects slopes of set sizes versus RTs, indicating that priming of motion direction affects the search process itself; in other words, the stage of processing affected by priming and the search are not independent processing stages (see Wolfe et al., 1989; more generally, Sternberg, 1969; see also Sigurdardottir et al., 2008, for some related findings). Motion is, of course, quite effective at capturing our attention under most conditions (Franconeri \& Simons, 2003; Hillstrom \& Yantis, 1994), but our results here indicate that such capture may be modulated by what has gone before, the previous motion direction, and the previous task requirements.

\section{Theoretical Accounts of Visual Attention}

Visual search is often thought to reflect the combined influence of bottom-up processes reflecting local fea- 
ture contrasts or "bottom-up" salience (see Julesz, 1981; Koch \& Ullman, 1985; Treisman \& Gelade, 1980), together with "top-down" or strategic processes that selectively activate pathways appropriate for the task at hand (Duncan \& Humphreys, 1989; Treisman \& Sato, 1990; Wolfe, 1994). "Pop-out" visual search has traditionally been thought to be based on contrasts such as background against foreground (He \& Nakayama, 1992; Kristjánsson, 2006c), luminance or color contrast (Theeuwes \& Kooi, 1994; Treisman \& Gelade, 1980; Wang et al., 2005; Wolfe, 1994), or shape differences (Kristjánsson \& Tse, 2001; Treisman \& Gormican, 1988; Wolfe, Yee, \& FriedmanHill, 1992). Such contrasts determine the way attention is allocated across the visual scene, in addition to the goals of the observer, or what is commonly referred to as top-down guidance (see Wolfe, 1994). Pop-out search should, by such accounts, operate in an efficient parallel manner and should not be affected by trial history, since the target is always quite salient on account of feature contrasts (e.g., Treisman \& Gelade, 1980). Maljkovic and Nakayama $(1994,1996)$ and several others since (Goolsby \& Suzuki, 2001; Hillstrom, 2000; Huang, Holcombe, \& Pashler, 2004; Kristjánsson, 2006b; Maljkovic \& Martini, 2005; Olivers \& Meeter, 2006; Theeuwes, Riemann, \& Mortier, 2006, to name a few) have, however, shown that such search can be influenced by repetition of particular properties (e.g., target color) across successive trials, in visual search tasks where a unique feature (e.g., singleton color) distinguishes the target from the distractors. This repetition affects search that should not be affected by such history effects, since the target should stand out regardless. One might think that an obvious explanation for such repetition effects would be that responding is simply facilitated. There have been several ways of dealing with this possibility, however. Keeping the response repetition independent of feature repetition (as in Experiments 2 and 3 here, and in Maljkovic \& Nakayama, 1994) rules out explaining the priming by appealing to repetition of response to account for these effects. Also, Sigurdardottir et al. (2008) have shown effects of feature repetition upon sensitivity (as measured with $d^{\prime}$ ) that are independent of criterion shifts in both single-feature and conjunction search tasks.

Evidence showing the importance of task repetition in visual search suggests that bottom-up contrasts and top-down guidance via task instructions cannot be the whole story, but that how we parse a given visual scene and what guides where we orient our attention is strongly influenced by what we have previously viewed - or has been of importance to us, if only a few moments before. Kristjánsson et al. (2002; see also Wang et al., 2005) argued that intertrial priming can even account for a large portion of effects usually attributed to top-down guidance in many theories of visual search (Duncan \& Humphreys, 1989; Treisman \& Sato, 1990; Wolfe, 1994). They found that search speed and accuracy on a visual search task for the item of odd color and orientation became similar, with only a few repetitions of the same target orientation, to what it was when the target orientation was always the same within a whole block of trials. The efficiency of such search where the target remained constant was in the past usually attributed to the effects of instruction (i.e., the effect of top-down guidance), but these results show that it can be accounted for by intertrial priming, at least in that context. It should be noted, however, that Wolfe et al. (2003) favor a somewhat different explanation for such results, calling such priming effects implicit top-down guidance, since they depend upon what the observer has learned (implicitly or explicitly) about the preceding trials and are not completely determined by the nature of the stimulus itself, but are dependent upon the "state" of the observer. As priming exerts its effects, the observer is biased toward the feature that mattered to the task at hand on a preceding trial or trials.

Of special note in the present study is the strong effect of repetition of distractor motion direction. Such importance of distractor set repetition, or context repetition, is consistent with some previous results, but is shown here for the first time to occur with stimuli defined by motion direction. Lamy et al. (2008) found that both distractor inhibition and target activation affect search, and also found that these effects were seemingly independent and additive. Kristjánsson and Driver $(2005,2008)$ observed similar results. They tested observers' performance during search for black or white filled or unfilled disks, independently varying target and distractor identity. They found that the effects of distractor repetition were at least as strong as those attributable to target repetition, and occurred independently of target repetition. This is also what we observed in the present experiments (in particular in Experiments 1A, 1B, 4, and 5).

Geyer et al. (2006) tested performance on a search for a target item of odd color and orientation relative to the distractors (based on the multiconjunction task introduced by Nakayama, Wang, \& Kristjansson, 2000; see also Kristjánsson et al., 2002; Wang et al., 2005), again independently varying target and distractor identity. Repetition benefits were the largest when both target and distractor identity were repeated, but the largest portion of this effect could be accounted for by distractor repetition. According to Geyer et al. (2006), distractor priming may even overshadow priming effects from target repetition under some conditions.

\section{Theoretical Accounts of Priming Effects in Vision}

Priming in visual search tasks has been explained by some in terms of increased saliency of primed features or facilitated deployment of attention to those features (e.g., Becker, 2008; Goolsby \& Suzuki, 2001; Kristjánsson, 2006a; Maljkovic \& Nakayama, 1994). Note that Ásgeirsson and Kristjánsson (2008) have made a strong counterargument to the case for the episodic retrieval account, suggesting that the results and implications from Huang et al. (2004) may not be as clear-cut as they argued. Kristjánsson, Ingvarsdóttir, and Teitsdóttir (2008) have then argued for a "hybrid" model of priming where different priming patterns are found for different object types. They contrasted visual search performance for objects that 
lend themselves differently well to object formation, and found that stimuli conducive to object-based processing required repetition of the whole object for priming to occur, whereas single-feature repetition was enough for priming to be seen for objects more likely to "fall apart" into their constituent features in perceptual processing (less conducive to object-based processing, in other words).

Such a hybrid model is supported by neuroimaging results of priming. Kristjánsson et al. (2007) tested priming for color and for position (see also Geng et al., 2006) in a variant of the diamond cutoff discrimination task introduced by Bravo and Nakayama (1992) and also used by Maljkovic and Nakayama (1994). They found that priming effects were correlated with activity in early visual areas, likely to reflect feature-based priming effects, whereas activity modulations correlated with priming were also seen for higher level areas, such as the frontoparietal attention network (see Corbetta \& Shulman, 2002; Desimone \& Duncan, 1995; Kastner \& Ungerleider, 2001; Ruff, Kristjánsson, \& Driver, 2007), that may reflect facilitated object-based processing with repetition. Finally, distinct effects were found in the fusiform gyrus when both the color of the target and its position were repeated; or, in other words, the whole display "gestalt" was repeated. Activity in this region may reflect priming of the global "gestalt" of the display - that is, when every feature is in the same place as on the previous trial.

Studies using lesioning or temporary lesioning with transcranial magnetic stimulation (TMS) also suggest that diverse cortical areas play a role in the priming patterns, depending on the particular primed feature or stimulus characteristic. One example is that lesions of V4 and TEO in monkeys abolish color priming effects (Walsh, le Mare, Blaimire, \& Cowey, 2000). Campana and colleagues have tested priming of motion direction concurrently with TMS. Disrupting the function of V5/MT in humans through temporal lesioning with TMS disrupts motion direction priming but not priming of color or spatial position (Campana, Cowey, \& Walsh, 2002, 2006). Selective disruption of frontal eye fields with TMS then strongly reduces priming of spatial position (Campana, Cowey, Casco, Oudsen, \& Walsh, 2007). All this suggests that a varied network of areas plays a role in the demonstrated behavioral priming patterns, supporting hybrid views of priming, where different types of priming occur for different types of objects or different features. The above evidence also suggests that neuroimaging studies of motion priming would be of great interest.

The present experiments are the first designed to obtain a thorough characterization of priming of motion direction in visual search. Strong priming effects from repetition of motion direction were found under a large number of conditions. Motion may thus with confidence be added to a list of features whose repetition in visual search tasks leads to facilitated attention shifts. The results also demonstrate the importance of the effects of the repetition of context (or distractor sets) in a motion direction visual search task; furthermore, the results add to previous evidence suggesting that priming in visual search has an effect on the search process itself, rather than affecting response selection or perceptual processes preceding the search. Results from a number of experimental paradigms have in recent years uncovered how important what occurs in the recent past appears to be for how we perceive and interpret the present (see Kristjánsson, 2006a). Our present results add to this experimental evidence by showing the importance of repetition of motion direction on targets as well as distractor sets for the way attention spreads across the visual field.

\section{AUTHOR NOTE}

This research was supported by grants from the research fund of the University of Iceland and the Human Frontiers Science Program. We thank Todd Horowitz, Joseph Krummenacher, Juan Lupiáñez, and Jeremy Wolfe for very helpful comments on previous versions of the manuscript. Correspondence concerning this article should be addressed to Á. Kristjánsson, Department of Psychology, University of Iceland, Oddi v. Sturlugötu, 101 Reykjavík, Iceland (e-mail: ak@hi.is).

\section{REFERENCES}

Anstis, S., Verstraten, F., \& Mather, G. (1998). The motion aftereffect. Trends in Cognitive Sciences, 2, 111-117.

Ásgeirsson, Á. G., \& KristuánsSON, Á. (2008). Episodic retrieval accounts of priming in visual search explain only a limited subset of findings on priming. Perception, 37(Suppl.), 93-94.

BECKER, S. I. (2008). The mechanism of priming: Episodic retrieval or priming of pop-out? Acta Psychologica, 127, 324-339.

Becker, S. I., \& Horstmann, G. (2009). A feature weighting account of priming in conjunction search. Attention, Perception, \& Psychophysics, 71, 258-272.

Bravo, M. J., \& Nakayama, K. (1992). The role of attention in different visual search tasks. Perception \& Psychophysics, 51, 465-472.

Bundesen, C. (1990). A theory of visual attention. Psychological Review, 97, 523-547.

Bundesen, C., \& Habekost, T. (2008). Principles of visual attention. Oxford: Oxford University Press.

Campana, G., Cowey, A., Casco, C., Oudsen, I., \& Walsh, V. (2007). Left frontal eye field remembers "where" but not "what." Neuropsychologia, 45, 2340-2345.

Campana, G., Cowey, A., \& Walsh, V. (2002). Priming of motion direction and area V5/MT: A test of perceptual memory. Cerebral Cortex, 12, 663-669.

Campana, G., Cowey, A., \& Walsh, V. (2006). Visual area V5/MT remembers "what" but not "where." Cerebral Cortex, 16, 1766-1770.

Campana, G., Pavan, A., \& Casco, C. (2008). Priming of first- and second-order motion: Mechanisms and neural substrates. Neuropsychologia, 46, 393-398.

Cavanagh, P., \& Mather, G. (1989). Motion: The long and short of it. Spatial Vision, 4, 103-129.

Chaudhuri, A. (1990). Modulation of the motion aftereffect by selective attention. Nature, 344, 60-62.

Chubb, C., \& Sperling, G. (1988). Drift-balanced random stimuli: A general basis for studying non-Fourier motion perception. Journal of the Optical Society of America A, 5, 1986-2007.

Chun, M. M., \& JiAng, Y. (1998). Contextual cueing: Implicit learning and memory of visual context guides spatial attention. Cognitive Psychology, 36, 28-71.

Clifford, C. W. G. (2002). Perceptual adaptation: Motion parallels orientation. Trends in Cognitive Sciences, 6, 136-143.

Clifford, C. W. G., \& Langley, K. (1996). Psychophysics of motion adaptation parallels insect electrophysiology. Current Biology, 6, 1340-1342.

Clifford, C. W. G., \& Wenderoth, P. (1999). Adaptation to temporal modulation can enhance differential speed sensitivity. Vision Research, 39, 4324-4332.

Comtois, R. (2003). Vision Shell PPC [Software libraries]. Cambridge, MA: Author. 
Corbetta, M., \& Shulman, G. L. (2002). Control of goal-directed and stimulus-driven attention in the brain. Nature Reviews Neuroscience, 3, 201-215.

Desimone, R., \& Duncan, J. (1995). Neural mechanisms of selective visual attention. Annual Review of Neuroscience, 18, 193-222.

Dick, M., Ullman, S., \& Sagi, D. (1987). Parallel and serial processes in motion detection. Science, 237, 400-402.

Duncan, J., \& Humphreys, G. W. (1989). Visual search and stimulus similarity. Psychological Review, 96, 433-458.

Egeth, H., Virzi, R., \& Garbart, H. (1984). Searching for conjunctively defined targets. Journal of Experimental Psychology: Human Perception \& Performance, 10, 32-39.

Found, A., \& MüLler, H. J. (1996). Searching for unknown feature targets on more than one dimension: Investigating a "dimensionweighting" account. Perception \& Psychophysics, 58, 88-101.

FranCONERI, S. L., \& SimONS, D. J. (2003). Moving and looming stimuli capture attention. Perception \& Psychophysics, 65, 999-1010.

Geng, J. J., Eger, E., Ruff, C., Kristuánsson, Á., Rotshtein, P., \& Driver, J. (2006). On-line attentional selection from competing stimuli in opposite visual fields: Effects on human visual cortex and control processes. Journal of Neurophysiology, 96, 2601-2612.

Geyer, T., Müller, H. J., \& Krummenacher, J. (2006). Cross-trial priming in visual search for singleton conjunction targets: Role of repeated target and distractor features. Perception \& Psychophysics, 68, 736-749.

Geyer, T., Müller, H. J., \& Krummenacher, J. (2007). Cross-trial priming of element positions in pop-out visual search is dependent on stimulus arrangement. Journal of Experimental Psychology: Human Perception \& Performance, 33, 788-797.

GiBson, J. J. (1937). Adaptation with negative aftereffect. Psychological Review, 44, 222-244

Goldstein, A. G. (1957). Judgments of visual velocity as a function of length of observation. Journal of Experimental Psychology, 54, 457-461.

GoolsBY, B. A., \& SuZUKI, S. (2001). Understanding priming of colorsingleton search: Roles of attention at encoding and "retrieval." Perception \& Psychophysics, 63, 929-944.

He, Z. J., \& NAKAYAMA, K. (1992). Surfaces vs. features in visual search. Nature, 359, 231-233.

Hillstrom, A. P. (2000). Repetition effects in visual search. Perception \& Psychophysics, 62, 800-817.

Hillstrom, A. P., \& Yantis, S. (1994). Visual motion and attentional capture. Perception \& Psychophysics, 55, 399-411.

Huang, L., Holcombe, A., \& Pashler, H. (2004). Repetition priming in visual search: Episodic retrieval, not feature priming. Memory \& Cognition, 32, 12-20.

JULESZ, B. (1981). A theory of preattentive texture discrimination based on first-order statistics of textons. Biological Cybernetics, 41, 131-138.

Kanai, R., \& Verstraten, F. A. (2005). Perceptual manifestations of fast neural plasticity: Motion priming, rapid motion aftereffect and perceptual sensitization. Vision Research, 45, 3109-3116.

KaStNer, S., \& Ungerleider, L. G. (2001). The neural basis of biased competition in human visual cortex. Neuropsychologia, 39, 12631276.

Koch, C., \& Ullman, S. (1985). Shifts in selective visual attention: Towards the underlying neural circuitry. Human Neurobiology, 4, 219-227.

KoHN, A. (2007). Visual adaptation: Physiology, mechanisms, and functional benefits. Journal of Neurophysiology, 97, 3155-3164.

KRISTJÁNSSON, A. (2001). Increased sensitivity to speed changes during adaptation to first-order, but not to second-order motion. Vision Research, 41, 1825-1832.

KRISTJÁnSSON, Á. (2006a). Rapid learning in attention shifts: A review. Visual Cognition, 13, 324-362.

KRISTJÁnsSON, Á. (2006b). Simultaneous priming along multiple dimensions in a visual search task. Vision Research, 46, 2554-2570.

KRISTJÁNSSON, Á. (2006c). Surface assignment modulates objectformation for visual short-term memory. Perception, 35, 865-881.

KRISTJÁnsSON, Á. (2008). "I know what you did on the last trial”: A selective review of research on priming in visual search. Frontiers in Bioscience, 13, 1171-1181.

KRISTJÁNSSON, Á. (2009). Independent and additive repetition prim- ing of motion direction and color in visual search. Psychological Research, 73, 158-166.

Kristjánsson, Á., \& Driver, J. (2005). Priming in vision: Target repetition effects, context effects and role reversal effects. Perception, 34(Suppl.), 40c.

KristJÁnSSON, Á., \& Driver, J. (2008). Priming in visual search: Separating the effects of target repetition, distractor repetition and rolereversal. Vision Research, 48, 1217-1232.

Kristjánsson, Á., IngVarsdóttir, Á., \& Teitsdóttir, U. D. (2008). Object-based and feature-based priming in visual search. Psychonomic Bulletin \& Review, 15, 378-384.

Kristuánsson, Á., \& NAKAYAMA, K. (2003). A primitive memory system for the deployment of transient attention. Perception \& Psychophysics, 65, 711-724.

Kristjánsson, A., \& Tse, P. U. (2001). Curvature discontinuities are cues for rapid shape analysis. Perception \& Psychophysics, 63, 390-403.

Kristuánsson, Á., Vuilleumier, P., Malhotra, P., Husain, M., \& Driver, J. (2005). Priming of color and position during visual search in unilateral spatial neglect. Journal of Cognitive Neuroscience, 17, 859-873.

Kristjánsson, Á., Vuilleumier, P., Schwartz, S., Macaluso, E., \& DRIVER, J. (2007). Neural basis for priming of pop-out revealed with fMRI. Cerebral Cortex, 17, 1612-1624.

Kristuánsson, A., Wang, D. L., \& Nakayama, K. (2002). The role of priming in conjunctive visual search. Cognition, 85, 37-52.

Kunar, M. A., Flusberg, S. J., Horowitz, T. S., \& Wolfe, J. M. (2007). Does contextual cuing guide the deployment of attention? Journal of Experimental Psychology: Human Perception \& Performance, 33, 816-828.

Lamy, D., Antebi, C., Aviani, N., \& Carmel, T. (2008). Priming of pop-out provides reliable measures of target activation and distractor inhibition in selective attention. Vision Research, 48, 30-41.

Ledgeway, T., \& Smith, A. T. (1997). Changes in perceived speed following adaptation to first-order and second-order motion. Vision Research, 37, 215-224.

LU, Z.-L., \& SPERLING, G. (1995). The functional architecture of human visual motion perception. Vision Research, 35, 2697-2722.

Magnussen, S., \& GReEnlee, M. W. (1999). The psychophysics of perceptual memory. Psychological Research, 62, 81-92.

Maljkovic, V., \& Martini, P. (2005). Implicit short-term memory and event frequency effects in visual search. Vision Research, 45, 28312846.

Maljkovic, V., \& Nakayama, K. (1994). Priming of pop-out: I. Role of features. Memory \& Cognition, 22, 657-672.

Maljkovic, V., \& Nakayama, K. (1996). Priming of pop-out: II. The role of position. Perception \& Psychophysics, 58, 977-991.

Maljkovic, V., \& NaKayama, K. (2000). Priming of pop-out: III. A short term implicit memory system beneficial for rapid target selection. Visual Cognition, 7, 571-595.

McLeod, P., Driver, J., \& CrisP, J. (1988). Visual search for a conjunction of movement and form is parallel. Nature, 332, 154-155.

Müller, H. J., Heller, D., \& Ziegler, J. (1995). Visual search for singleton feature targets within and across feature dimensions. Perception \& Psychophysics, 57, 1-17.

Müller, H. J., Krummenacher, J., \& Heller, D. (2004). Dimensionspecific intertrial facilitation in visual search for pop-out targets: Evidence for a top-down modulable visual short-term memory effect. Visual Cognition, 11, 577-602.

Müller, H. J., Riemann, B., \& Krummenacher, J. (2003). Visual search for singleton feature targets across dimensions: Stimulusand expectancy-driven effects in dimensional weighting. Journal of Experimental Psychology: Human Perception \& Performance, 29, 1021-1035.

NaKayama, K., \& Silverman, G. H. (1986). Serial and parallel processing of visual feature conjunctions. Nature, 320, 264-265.

Nakayama, K., Wang, D. L., \& Kristuánsson, Á. (2000). Efficient visual search not explained by local feature mechanisms or top-down guidance. Investigative Ophthalmology \& Vision Science, 41, S759.

Olivers, C. N. L., \& Humphreys, G. W. (2003). Attentional guidance by salient feature singletons depends on intertrial contingencies. Journal of Experimental Psychology: Human Perception \& Performance, 29, 650-657. 
Olivers, C. N. L., \& Meeter, M. (2006). On the dissociation between compound and present/absent tasks in visual search: Intertrial priming is ambiguity-driven. Visual Cognition, 13, 1-28.

Olivers, C. N. L., \& Meeter, M. (2008). Feature priming in visual search does not depend on the dimensional context. Visual Cognition, 16, 785-803.

Ruff, C. C., Kristuánsson, Á., \& Driver, J. (2007). Readout from iconic memory involves similar neural processes as selective spatial attention. Psychological Science, 18, 901-909.

Saevarsson, S., Jóelsdóttir, S., Hualtason, H., \& Kristuánsson, Á. (2008). Repetition of distractor sets improves visual search performance in hemispatial neglect. Neuropsychologia, 45, 1161-1169.

Shibuya, H., \& Bundesen, C. (1988). Visual selection from multielement displays: Measuring and modeling effects of exposure duration. Journal of Experimental Psychology: Human Perception \& Performance, 14, 591-600.

Sigurdardottir, H. M., Kristuánsson, Á., \& Driver, J. (2008). Repetition streaks increase perceptual sensitivity in brief visual search displays. Visual Cognition, 16, 643-658.

STERNBERG, S. (1969). The discovery of processing stages: Extension of Donders' method. Acta Psychologica, 30, 276-315.

Theeuwes, J., \& KooI, F. L. (1994). Parallel search for a conjunction of contrast polarity and shape. Vision Research, 34, 3013-3016.

Theeuwes, J., Riemann, B., \& Mortier, K. (2006). Visual search for featural singletons: No top-down modulation, only bottom-up priming. Visual Cognition, 14, 466-489.

Thompson, P. (1981). Velocity aftereffects: The effects of adaptation to moving stimuli on the perception of subsequently seen moving stimuli. Vision Research, 21, 337-345.

Treisman, A. (1988). Features and objects: The fourteenth Bartlett memorial lecture. Quarterly Journal of Experimental Psychology, 40A, 201-237.

Treisman, A. (1992). Perceiving and re-perceiving objects. American Psychologist, 47, 862-875.

Treisman, A., \& Gelade, G. (1980). A feature-integration theory of attention. Cognitive Psychology, 12, 97-136.

Treisman, A., \& Gormican, S. (1988). Feature analysis in early vision: Evidence from search asymmetries. Psychological Review, 95, 15-48.

Treisman, A., \& Sato, S. (1990). Conjunction search revisited. Journal of Experimental Psychology: Human Perception \& Performance, 16, 459-478.
Walsh, V., le Mare, C., Blaimire, A., \& Cowey, A. (2000). Normal discrimination performance is accompanied by priming deficits in monkeys with V4 or TEO lesions. NeuroReport, 11, 1459-1462.

Wang, D. L., Kristuánsson, Á., \& Nakayama, K. (2005). Efficient visual search without top-down or bottom-up guidance. Perception \& Psychophysics, 67, 239-253.

Weidner, R., Pollmann, S., Müller, H. J., \& von Cramon, D. Y. (2002). Top-down controlled visual dimension weighting: An eventrelated fMRI study. Cerebral Cortex, 12, 318-328.

Wolfe, J. M. (1994). Guided Search 2.0: A revised model of visual search. Psychonomic Bulletin \& Review, 1, 202-238.

Wolfe, J. M., Butcher, S. J., LeE, C., \& Hyle, M. (2003). Changing your mind: On the contributions of top-down and bottom-up guidance in visual search for feature singletons. Journal of Experimental Psychology: Human Perception \& Performance, 29, 483-502.

Wolfe, J. M., Cave, K. R., \& Franzel, S. L. (1989). Guided search: An alternative to the feature integration model for visual search. Journal of Experimental Psychology: Human Perception \& Performance, 15, 419-433.

Wolfe, J. M., Friedman-Hill, S. R., \& Chun, M. M. (1991). A “sameness" operation in visual search. Investigative Ophthalmology \& Visual Science, 32, 715

Wolfe, J. M., Yee, A., \& Friedman-Hill, S. R. (1992). Curvature is a basic feature for visual search tasks. Perception, 21, 465-480.

\section{NOTES}

1. As opposed to motion defined by second-order properties such as contrast differences (see Cavanagh \& Mather, 1989; Chubb \& Sperling, 1988; Kristjánsson, 2001; Ledgeway \& Smith, 1997; Lu \& Sperling, 1995).

2. In Experiments 3, 4, and 5, some of the Gabor patches were redscale or greenscale, and these brightness values are specified in those respective Method sections.

3. All reported $p$ values are one-tailed, and the critical $\alpha$ levels are Bonferroni corrected.

4. We are grateful to Joseph Krummenacher for pointing out this possible interpretation of the foregoing experiments.

(Manuscript received February 14, 2008; revision accepted for publication January 31, 2009.) 